Pathophysiology of Lipid Peroxides and Their 2nd Products

Guest Editor: Kenji Fukuzawa

\title{
Introduction to Serial Reviews: \\ Pathophysiology of Lipid Peroxides and Their 2-nd Products
}

\author{
Kenji Fukuzawa \\ Faculty of Pharmaceutical Sciences, University of Tokushima, Tokushima, Japan
}

Recently, more attention has been given to the pathophysiology of lipid peroxides, especially to that of 2 nd products derived from them because of their biological activities and high reactivity to biological substances in pathogenesis of many diseases.

Accordingly, the editor organized the symposium entitled "Recent Advances on Pathophysiology of Lipid Peroxides" in the 26th Biannual Meeting of the Japanese Society of Lipid Peroxide and Free Radical Research, which was held on October 31 and November 1, 2002, in Tokushima city, Japan. The Serial Review was planed out by the editor, who asked to the contributors to present their review papers following the symposium.

This set of Serial Reviews will be contributed to the progress of the research on the pathophysiology of lipid peroxides and their degradation products, and also to the improvement of the prevention of their-related diseases. 